\title{
Nonrenormalization and Operator Mixing via On-Shell Methods
}

\author{
Zvi Bern, ${ }^{1,2}$ Julio Parra-Martinez, ${ }^{1}$ and Eric Sawyer ${ }^{1}$ \\ ${ }^{1}$ Mani L. Bhaumik Institute for Theoretical Physics, UCLA Department of Physics and Astronomy, Los Angeles, California 90095, USA \\ ${ }^{2}$ Theoretical Physics Department, CERN, 1211 Geneva 23, Switzerland
}

(Received 22 October 2019; accepted 20 November 2019; published 4 February 2020)

\begin{abstract}
Using on-shell methods, we present a new perturbative nonrenormalization theorem for operator mixing in massless four-dimensional quantum field theories. By examining how unitarity cuts of form factors encode anomalous dimensions, we show that longer operators are often restricted from renormalizing shorter operators at the first order where Feynman diagrams exist. The theorem applies quite generally and depends only on the field content of the operators involved. We apply our theorem to operators of dimension five through seven in the standard model effective field theory, including examples of nontrivial zeros in the anomalous-dimension matrix at one through four loops. The zeros at two and higher loops go beyond those previously explained using helicity selection rules. We also include explicit sample calculations at two loops.
\end{abstract}

DOI: 10.1103/PhysRevLett.124.051601

Introduction.-A key challenge in particle physics is to identify physics beyond the standard model. Because current experimental data at colliders are well described by the standard model, it is unclear which theoretical direction will ultimately prove to be the one chosen by nature. Therefore, it is important to quantify new physics beyond the standard model in a systematic, modelindependent manner. The theoretical framework for doing so is via effective field theories that extend the standard model Lagrangian by adding higher-dimension operators $[1,2]$

$$
\Delta \mathcal{L}=\sum_{i} c_{i} \mathcal{O}_{i}
$$

with coefficients $c_{i}$ suppressed by powers of a high-energy scale $\Lambda$ dictated by the dimension of $\mathcal{O}_{i}$. The resulting theory, known as the standard model effective field theory (SMEFT), is reviewed in Ref. [3].

As for all quantum field theories, renormalization induces mixing of these operators. This can be parametrized by the renormalization group equation

$$
16 \pi^{2} \frac{\partial c_{i}}{\partial \log \mu}=\gamma_{i j}^{\mathrm{UV}} c_{j},
$$

where $\gamma_{i j}^{\mathrm{UV}}$ is the anomalous-dimension matrix and $\mu$ is the renormalization scale. Usually, $\gamma_{i j}^{\mathrm{UV}}$ is calculated

Published by the American Physical Society under the terms of the Creative Commons Attribution 4.0 International license. Further distribution of this work must maintain attribution to the author(s) and the published article's title, journal citation, and DOI. Funded by SCOAP ${ }^{3}$. perturbatively in the marginal couplings of the standard model Lagrangian, which we will denote collectively as $g$. The complete one-loop anomalous-dimension matrix for operators up to dimension six has been computed in Refs. [4,5]. These calculations reveal a number of vanishing entries related to supersymmetry [6], which seem surprising at first because there are valid diagrams that can be written down. These zeros have been elegantly explained [7] using tree-level helicity selection rules [8], which set certain classes of tree-level amplitudes to zero. The tree-level vanishings imply, through unitarity, that certain logarithms and their associated anomalous dimensions are not present. Although these selection rules are reminiscent of supersymmetric ones, they hold for generic massless quantum field theories in four dimensions.

Might it be possible that beyond one loop there are new nontrivial zeros? At first sight, this seems rather unlikely because the helicity selection rules fail to hold at loop level. In this Letter, we show that, contrary to expectations, there are, in fact, additional nontrivial zeros in the higher-loop anomalous-dimension matrix. As in Ref. [7], our only assumption is that the theory does not contain any relevant couplings (e.g., masses). To state the new nonrenormalization theorem, we define the length of an operator, $l(\mathcal{O})$, as the number of fundamental field insertions in $\mathcal{O}$. Then the statement of the theorem is as follows.

Theorem 1.-Consider operators $\mathcal{O}_{s}$ and $\mathcal{O}_{l}$ such that $l\left(\mathcal{O}_{l}\right)>l\left(\mathcal{O}_{s}\right) . \mathcal{O}_{l}$ can renormalize $\mathcal{O}_{s}$ at $L$ loops only if $L>l\left(\mathcal{O}_{l}\right)-l\left(\mathcal{O}_{s}\right)$.

At fixed loop order, sufficiently long operators cannot renormalize short operators because there would be too many legs to form a diagram with the right structure. Such zeros in the anomalous-dimension matrix are trivial. As written above, the theorem applies nontrivially at 
$\left[l\left(\mathcal{O}_{l}\right)-l\left(\mathcal{O}_{s}\right)\right]$ loops, i.e., the first loop order at which there could be renormalization because diagrams exist. However, in a general theory with multiple types of fields, the first renormalization can be delayed even further, depending on the precise field content of the two operators. We encapsulate this into the more general rule:

Theorem 2.-If at any given loop order, the only diagrams for a matrix element with the external particle content of $\mathcal{O}_{s}$ but an insertion of $\mathcal{O}_{l}$ involve scaleless bubble integrals, there is no renormalization of $\mathcal{O}_{s}$ by $\mathcal{O}_{l}$.

What makes them nontrivial is that Feynman diagrams exist that seem as if they should contribute to an anomalous dimension, but fail to do so because the diagrams do not generate the appropriate logarithms. The Feynman-diagram language can obscure this because individual diagrams are not gauge invariant. While not difficult to disentangle at one loop, at higher loops, it becomes more advantageous to work in an on-shell formalism, which only takes gaugeinvariant quantities as input. Indeed, modern unitarity methods [9] have clarified the structure of loop amplitudes resulting in significant computational advantages for a variety of problems, including the computation of form factors and associated anomalous dimensions [10].

Renormalization and form factors.-Traditionally, the anomalous dimension corresponding to the renormalization of an operator $\mathcal{O}_{i}$ by an operator $\mathcal{O}_{j}$ is extracted from UV divergences. These can be found, for instance, in form factors

$$
F_{j}\left[p_{1}, \ldots, p_{n} ; q ; \mu\right]=\left\langle p_{1}, \ldots, p_{n}\left|\mathcal{O}_{j}(q)\right| 0\right\rangle,
$$

with an operator insertion $\mathcal{O}_{j}$ and external states $\left|p_{1}, \ldots, p_{n}\right\rangle$ that overlap with states created by $\mathcal{O}_{i}$. The divergences and associated anomalous dimensions can also be obtained from one-particle irreducible effective actions or from scattering amplitudes, $\mathcal{M}_{\mathcal{O}_{j}}$, corresponding to form factors with the operator momentum injection $q$ set to zero.

Here, we use the elegant on-shell approach developed by Caron-Huot and Wilhelm that extracts anomalous dimensions directly from renormalized quantities [11]. In this approach, the intuition that the renormalization properties of the theories are encoded in on-shell form factors through their logarithms is made precise by the following equation:

$$
e^{-i \pi D} F^{*}=S F^{*},
$$

where $F^{*}$ is the conjugate form factor with an insertion of an $\mathcal{O}_{j}$ operator. This relates the phase of the $S$-matrix, $S$, to the dilatation operator $D$ which extracts anomalous dimensions. We point the interested reader to Ref. [11] for its derivation.

For simplicity, we use dimensional regularization. In this case, the dilatation operator $D$ is related to the single renormalization scale $\mu=\mu_{\mathrm{UV}}=\mu_{\mathrm{IR}}$, as $\quad D \simeq-\mu \partial_{\mu}$. Expanding Eq. (4) at one loop one obtains the following description of the renormalization of $\mathcal{O}_{i}$ by $\mathcal{O}_{j}$ :

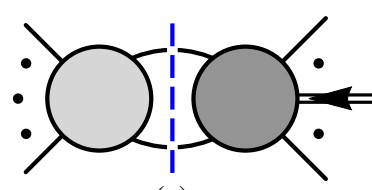

(a)

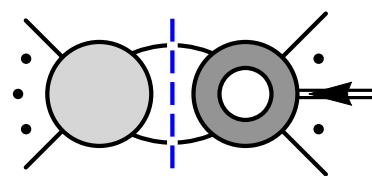

(c)

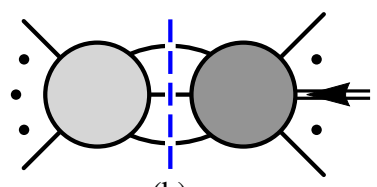

(b)

(d)

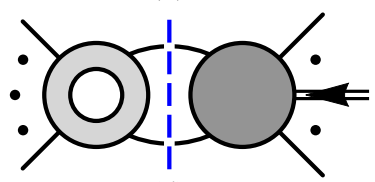

FIG. 1. Unitarity cuts relevant for the extraction of anomalous dimensions from one- (a) and two-loop (b-d) form factors.. The darker blobs indicate a higher-dimension operator insertion. The double-lined arrow indicates the insertion of additional off-shell momentum from the operator. The dashed line indicates the integral over phase space of the particles crossing the cut.

$$
\begin{gathered}
{\left[\gamma_{i j}^{\mathrm{UV}}-\gamma_{i j}^{\mathrm{IR}}+\beta(g) \partial_{g}\right]^{(1)}\left\langle p_{1}, \ldots, p_{n}\left|\mathcal{O}_{i}\right| 0\right\rangle^{(0)}} \\
=-\frac{1}{\pi}\left\langle p_{1}, \ldots, p_{n}\left|M \otimes \mathcal{O}_{j}\right| 0\right\rangle .
\end{gathered}
$$

On the left hand side, we find the tree-level form factor of $\mathcal{O}_{i}$, the beta function $\beta(g)$ of the couplings $g$, the anomalous dimensions $\gamma_{\mathrm{UV}}$, which are the objects of interest, and the infrared anomalous dimensions $\gamma_{\mathrm{IR}}$, which arise from soft and/or collinear logarithms. The superscripts denote the perturbative order. The right hand side arises from the term $\mathcal{M} F^{*}$, where $\mathcal{M}=-i(S-1)$ is the scattering amplitude. The notation $\otimes$ here refers to an integration over the phase space of intermediate two-particle states in the product. This simply corresponds to a one-loop unitarity cut, as depicted in Fig. 1(a). Schematically, Eq. (5) says that, up to terms coming from the $\beta$ function, one-loop anomalous dimensions are eigenvalues of the $S$-matrix, with the form factors being the corresponding eigenvectors. More practically, this equation describes how to systematically extract the anomalous dimensions from the coefficients of logarithms by taking discontinuities of the form factor.

The fact that dependence on the renormalization scale is related to discontinuities in kinematic variables is no surprise since the arguments of logarithms must balance kinematic variables against the renormalization scale to make them dimensionless. This observation has also been used to efficiently determine the renormalization-scale dependence of the two-loop counterterm in pure Einstein gravity from unitarity [12].

At higher-loop orders, other unitarity cuts, matching the order of the anomalous dimension, need to be considered. For instance, at two loops, the three-particle cut is required, as well as the two-particle cut between the tree-level amplitude and the one-loop form factor and vice versa, as in Figs. 1(b)-1(d).

Nonrenormalization theorem.-We would like to consider the renormalization of a shorter operator $\mathcal{O}_{s}$ by a 
longer operator $\mathcal{O}_{l}$. This could be, for example, the renormalization of $\phi^{2} F^{2}$ by $\phi^{6}$, where $\phi$ is a scalar and $F$ is a vector field strength. For simplicity, we will take $\mathcal{O}_{s}$ and $\mathcal{O}_{l}$ to be single operators, though in general, they represent collections of operators with the same field content but differing Lorentz contractions or color factors. Because our arguments rely only on the field content and basic structure of the unitarity cuts, our conclusions will apply just as well to the more general case.

The formalism reviewed above allows us to connect the anomalous dimensions to unitarity cuts of form factors, given knowledge of the $\beta$ function of the leading couplings and the infrared anomalous dimensions. Now, we show that, for the leading contributions, there is an even more direct connection between the ultraviolet anomalous dimensions and unitarity cuts.

The appearance of the $\beta$ function in Eq. (5) is avoided simply by extracting the anomalous dimensions from the minimal form factor of $\mathcal{O}_{s}$, which is defined as the one with the minimum number of legs needed to match the operator. We will denote this by a subscript on the state, $\left|p_{1}, \ldots, p_{n}\right\rangle_{s}$. Because of its defining property, the minimal tree-level form factor is local and does not depend on the couplings, $g$. Therefore, the dependence of the higher-loop analog of Eq. (5) on the $\beta$ function drops out.

Next, we would need knowledge of the infrared anomalous dimension $\gamma_{\mathrm{IR}}$. Infrared singularities are very well understood [13-16]. Our case is special, with a rather simple infrared structure. We are interested in the first loop order at which the higher-dimension operator could be renormalized. This would be the first loop order for which it is possible to write down valid diagrams. The lack of diagrams at lower-loop order means there cannot be any $\log \left(\mu_{\mathrm{IR}}\right)$ terms or corresponding $\gamma_{\mathrm{IR}}$ at the given loop order under consideration. In addition, infrared singularities are diagonal for the operators with distinct fields, mixing only via color. Therefore, at this order, $\gamma_{\mathrm{IR}}=0$. Various examples will be given in Ref. [17].

Thus, application of Eq. (4) is particularly simple for our case so that the relation between the first potentially nonvanishing anomalous dimension and unitarity cuts is direct

$$
\begin{aligned}
\left(\gamma_{s l}^{\mathrm{UV}}\right)^{(L)}{ }_{s}\left\langle p_{1}, \ldots, p_{n}\left|\mathcal{O}_{s}\right| 0\right\rangle^{(0)} & \\
\quad & =-\frac{1}{\pi}{ }_{s}\left\langle p_{1}, \ldots, p_{n}\left|\mathcal{M} \otimes \mathcal{O}_{l}\right| 0\right\rangle .
\end{aligned}
$$

With this relation at hand, it is now straightforward to argue for new nonrenormalization zeros by analyzing the allowed unitarity cuts. Equation (6) gives $\left(\gamma_{s l}^{\mathrm{UV}}\right)^{(L)}$ in terms of a sum over cuts of the form illustrated in Fig. 1. The left-hand side of any such $k$-particle cut is a $n_{\mathcal{M}}$-point amplitude, with the number of particles external to the cut equal to $n_{\mathcal{M}}-k$. Similarly, the right-hand side is an $n_{F}$-point form factor, with $n_{F}-k$ particles external to the cut. Now, for the minimal form factor, the total number of external particles must match the length of $\mathcal{O}_{s}$, so we must have the relation

$$
n_{\mathcal{M}}+n_{F}-2 k=l\left(\mathcal{O}_{s}\right) .
$$

The number of legs $n_{\mathcal{M}}$ and $n_{F}$ are both bounded from below. For the unitarity cut to be nonzero, the scattering amplitude on the left must have at least two external particles, that is, $n_{\mathcal{M}} \geq k+2$. On the other side, $n_{F}$ is restricted by the requirement that the form factor not include any scaleless bubbles. Since all legs of the form factor, including those crossing the cut, are on shell, any such scaleless bubbles would evaluate to zero. At one loop, for example, this implies $n_{F} \geq l\left(\mathcal{O}_{l}\right)$, which is the same as the tree level relation. At higher loops, the particle count can be reduced depending on the number of loops in the form factor, which produces the relation

$$
n_{F} \geq l\left(\mathcal{O}_{l}\right)-\left(L_{F}-1\right)-\delta_{L_{F}, 0} .
$$

Here, $L_{F}$ is the number of loops contained in the form factor. $\delta_{L_{F}, 0}$ is unity if the form factor is at tree level and zero, otherwise, which accounts for the fact that there is no reduction in particle number between tree level and one loop. By considering the possible placings of the loops in the cut or on either side of the cut, we have $L_{F} \leq L-(k-1)$, implying $n_{F} \geq l\left(\mathcal{O}_{l}\right)-L+k-\delta_{L_{F}, 0}$. Combining this with the condition on $n_{\mathcal{M}}$ and plugging in to equation (7), we obtain

$$
l\left(\mathcal{O}_{l}\right)-L+2-\delta_{L_{F}, 0} \leq l\left(\mathcal{O}_{s}\right) .
$$

This inequality shows that the difference in length of the operators can preclude the renormalization unless

$$
L>l\left(\mathcal{O}_{l}\right)-l\left(\mathcal{O}_{s}\right)
$$

and, thus, completes the proof of the first form of our theorem. In summary, we have shown that, at loop orders less than or equal to $l\left(\mathcal{O}_{l}\right)-l\left(\mathcal{O}_{s}\right)$, there are no allowed unitarity cuts that can capture the coefficient of $\log \left(\mu^{2}\right)$, which, in turn, implies that $\gamma_{s l}^{\mathrm{UV}}=0$. Equation (9) also shows that the contributions to the anomalous dimension at loop order $L=l\left(\mathcal{O}_{l}\right)-l\left(\mathcal{O}_{s}\right)+1$ are captured by cuts of the type in Figs. 1(a) and 1(b), that are given purely in terms of tree-level matrix elements. Cuts of the type in Fig. 1(c) are directly ruled out by Eq. (9), and cuts of the type in Fig. 1(d) are ruled out because $l\left(O_{l}\right)-l\left(O_{s}\right)+2$ legs need to be sewn across the cut to have a total of $l\left(O_{s}\right)$ external legs, so that all $l\left(O_{l}\right)-l\left(O_{s}\right)+1$ loops are accounted for in the cut. This observation should help in their computation, for instance, by allowing the use of four-dimensional helicity methods to evaluate the cut. It also implies that helicity selection rules can be active beyond one loop, contrary to expectations. 
TABLE I. Application of the nonrenormalization theorem to dimension-five operators. The operators labeling the rows are renormalized by the operators labeling the columns. $\times_{L}$ indicates the theorem applies at $L$-loop order. $(L)$ denotes that there are no diagrams before $L$ loops, but renormalization is possible at that order, since the required cuts can exist. Light-gray shading indicates a zero at one loop due to helicity selection rules, while dark-gray shading indicates the entry is a new zero predicted by our nonrenormalization theorem.

\begin{tabular}{l|c|c|c|c}
\hline \hline & $F^{2} \phi$ & $F \psi^{2}$ & $\phi^{2} \psi^{2}$ & $\phi^{5}$ \\
\hline$F^{2} \phi$ & & & $(2)$ & $\times_{2}$ \\
\hline$F \psi^{2}$ & & & $\times_{1}$ & $\times_{3}$ \\
\hline$\phi^{2} \psi^{2}$ & & & & $(2)$ \\
\hline$\phi^{5}$ & & & & \\
\hline \hline
\end{tabular}

Depending on the particle contents of the two operators, it might happen that there are no allowed unitarity cuts even at a higher loop order than the one predicted by the first form of the theorem. Instead of analyzing the unitarity cuts, this can be explained in the more familiar diagrammatic language. Clearly, if the only diagrams that can be drawn involve scaleless bubbles, there will be no available cut where all loops are included in the cut. Thus, diagrams with fewer cut legs will force the form factor to include the scaleless bubble and, thus, to evaluate to zero. Then, the corresponding anomalous dimension must also be zero. This explains the more general rule presented in the introduction. As noted above, this relies on the absence of infrared singularities whenever corresponding lowerloop form factors vanish.

Examples of zeros in the SMEFT at one loop are the renormalization of $F^{3}$ by $\phi^{2} F^{2}$, and of $D^{2} \phi^{4}, F \phi \psi^{2}$, and $D \phi^{2} \psi^{2}$ by $\phi^{3} \psi^{2}$, which were already explained using the helicity selection rules [7] but also follow from the principles described here. In contrast to the helicity

TABLE II. Application of the nonrenormalization theorem to dimension six. The notation is explained in Table I.

\begin{tabular}{l|c|c|c|c|c|c|c|c}
\hline \hline & $F^{3}$ & $\phi^{2} F^{2}$ & $F \phi \psi^{2}$ & $D^{2} \phi^{4}$ & $D \phi^{2} \psi^{2}$ & $\psi^{4}$ & $\phi^{3} \psi^{2}$ & $\phi^{6}$ \\
\hline$F^{3}$ & & $\times_{1}$ & $(2)$ & $\times_{2}$ & $\times_{2}$ & $\times_{2}$ & $\times_{3}$ & $\times_{3}$ \\
\hline$\phi^{2} F^{2}$ & & & & & & & $(2)$ & $\times_{2}$ \\
\hline$F \phi \psi^{2}$ & & & & & & & $\times_{1}$ & $\times_{3}$ \\
\hline$D^{2} \phi^{4}$ & & & & & & & $\times_{1}$ & $\times_{2}$ \\
\hline$D \phi^{2} \psi^{2}$ & & & & & & & $\times_{1}$ & $(3)$ \\
\hline$\psi^{4}$ & & & & & & & $(2)$ & $(4)$ \\
\hline$\phi^{3} \psi^{2}$ & & & & & & & & $(2)$ \\
\hline$\phi^{6}$ & & & & & & & & \\
\hline \hline
\end{tabular}

selection rules, however, our theorem can also apply at higher loops. The full set of zeros predicted by our rules for operators of dimensions five, six, and seven includes examples at one through four loops and is described in Tables I, II, and III, respectively. The tables also indicate the overlap between our theorem and the one-loop helicity selection rules of [7]. Note, we have combined some of the categories of operators of [7], since our theorem does not need to distinguish operators based on their chirality.

Two-loop examples.-Now, consider two calculations that show explicit examples, from Table II, of the nontrivial zeros in the anomalous-dimension matrix at two loops. The examples will also demonstrate the vanishing of $\gamma_{\mathrm{IR}}$. The first example is the renormalization of $\mathcal{O}_{\phi^{2} F^{2}}$ by $\mathcal{O}_{\phi^{6}}$, which is the entry $(2,8)$ of Table II.

The minimal two-loop form factor for $\mathcal{O}_{\phi^{2} F^{2}}$ includes two external scalars and two external gauge bosons. The product $\mathcal{M} F^{*}$ in Eq. (4) at two loops requires either a cut between a five-point amplitude and the tree-level form factor or a four-point amplitude and a one-loop form factor with an insertion of $\mathcal{O}_{\phi^{6}}$. However, the cut between the fivepoint amplitude and the tree-level form factor leaves five total external legs and, thus, cannot match the minimal form factor for $\mathcal{O}_{\phi^{2} F^{2}}$. For the cut between the four-point amplitude and the one-loop form factor to match the minimal form factor for $\mathcal{O}_{\phi^{2} F^{2}}$, the one-loop form factor would have to involve a massless tadpole, which would evaluate to zero.

We can also directly check that the (single) diagramFig. 2(a)-for the $\mathcal{O}_{\phi^{6}} \rightarrow \mathcal{O}_{\phi^{2} F^{2}}$ renormalization evaluates

TABLE III. Application of the nonrenormalization theorem to dimension seven. The notation is explained in Table I. The shortest and longest operators have been dropped from the list of columns and rows, respectively, since our theorem requires a reduction in length of the operators.

\begin{tabular}{l|c|c|c|c|c|c|c}
\hline \hline & $\phi^{3} F^{2}$ & $D^{2} \phi^{5}$ & $D \phi^{3} \psi^{2}$ & $\phi \psi^{4}$ & $F \phi^{2} \psi^{2}$ & $\phi^{4} \psi^{2}$ & $\phi^{7}$ \\
\hline$F^{3} \phi$ & $\times_{1}$ & $\times_{2}$ & $\times_{2}$ & $\times_{2}$ & $(2)$ & $\times_{3}$ & $\times_{3}$ \\
\hline$D^{2} F \phi^{3}$ & $\times_{1}$ & $\times_{1}$ & $\times_{1}$ & $\times_{2}$ & $\times_{1}$ & $\times_{2}$ & $\times_{3}$ \\
\hline$D F \phi \psi^{2}$ & $(2)$ & $\times_{2}$ & $\times_{1}$ & $\times_{1}$ & $\times_{1}$ & $\times_{2}$ & $\times_{4}$ \\
\hline$F^{2} \psi^{2}$ & $(2)$ & $(3)$ & $(2)$ & $(2)$ & $\times_{1}$ & $\times_{2}$ & $\times_{4}$ \\
\hline$D^{2} \phi^{2} \psi^{2}$ & $(2)$ & $(2)$ & $\times_{1}$ & $\times_{1}$ & $\times_{1}$ & $\times_{2}$ & $(4)$ \\
\hline$D \psi^{4}$ & $(3)$ & $(3)$ & $(2)$ & $\times_{1}$ & $(2)$ & $(3)$ & $(5)$ \\
\hline$\phi^{3} F^{2}$ & & & & & & $(2)$ & $\times_{2}$ \\
\hline$D^{2} \phi^{5}$ & & & & & & $\times_{1}$ & $\times_{2}$ \\
\hline$D \phi^{3} \psi^{2}$ & & & & & & $\times_{1}$ & $(3)$ \\
\hline$\phi \psi^{4}$ & & & & & & $(2)$ & $(4)$ \\
\hline$F \phi^{2} \psi^{2}$ & & & & & & $\times_{1}$ & $\times_{3}$ \\
\hline$\phi^{4} \psi^{2}$ & & & & & & & $(2)$ \\
\hline \hline
\end{tabular}




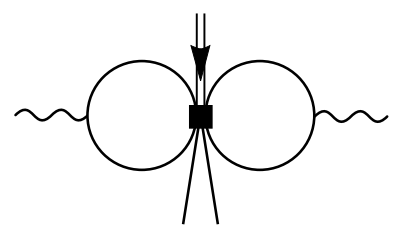

(a)

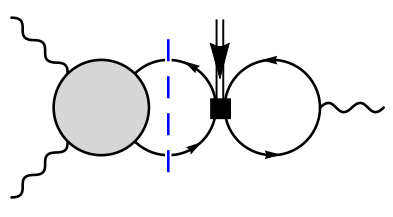

(b)
FIG. 2. (a) Diagram showing the only possible two loop contribution to the renormalization of $\mathcal{O}_{\phi^{2} F^{2}}$ by $\mathcal{O}_{\phi^{6}}$. (b) Cut of a form factor showing that $\mathcal{O}_{\psi^{4}}$ cannot renormalize $\mathcal{O}_{F^{3}}$ at two loops. The solid square indicates the insertion of the $\phi^{6}$ or $\psi^{4}$ operator, respectively.

to zero. By incorporating an IR regulator $\lambda_{\mathrm{IR}}$, we can evaluate the integral while keeping the UV and IR dependences separate and determine the behavior of the form factor in the limit $\lambda_{\mathrm{IR}} \rightarrow 0$. The integral for this diagram immediately factorizes, and each of the two loop integrals is of the form

$$
\int \frac{d^{D} \ell_{1}}{(2 \pi)^{D}} \frac{\left(2 \ell_{1}^{v}-k_{1}^{v}\right) \varepsilon_{1}^{v}}{\left(\ell_{1}^{2}-\lambda_{\mathrm{IR}}\right)\left[\left(\ell_{1}-k_{1}\right)^{2}-\lambda_{\mathrm{IR}}\right]} .
$$

This integral vanishes by the on-shell condition $k_{1}{ }^{v} \varepsilon_{1}{ }^{v}=0$ and Lorentz invariance, since $k_{1}$ is the only available momentum. Therefore, $\mathcal{O}_{\phi^{6}}$ cannot renormalize $\mathcal{O}_{\phi^{2} F^{2}}$ at two loops.

For a slightly more complex example, consider the renormalization of $\mathcal{O}_{F^{3}}$ by $\mathcal{O}_{\psi^{4}}$ at two loops, corresponding to entry $(1,6)$ of Table II. Again, for this process, the threeparticle cut between the five-point amplitude and the treelevel form factor does not produce the correct externalparticle state corresponding to the field content of $\mathcal{O}_{F^{3}}$. The two-particle cut between the four-point amplitude and the one-loop form factor with an insertion of $\mathcal{O}_{\psi^{4}}$ is shown in Fig. 2(b). By again adding an IR regulator, the result can be written as

$$
\int d \operatorname{LIPS}_{\ell_{1}} \frac{d^{D} \ell_{2}}{(2 \pi)^{D}} \frac{\operatorname{Tr}\left[X\left(\ell_{1}\right) \not \ell_{2} \phi_{3}\left(\not_{2}-\not \ell_{3}\right)\right]}{\left(\ell_{2}^{2}-\lambda_{\mathrm{IR}}\right)\left[\left(\ell_{2}-k_{3}\right)^{2}-\lambda_{\mathrm{IR}}\right]},
$$

where $X$ receives contributions from the multiple possible diagrams of the four-point amplitude and includes the remaining propagators. LIPS indicates integration over the Lorentz-invariant phase space of the particles crossing the cuts.

One can reduce the $\ell_{2}$ tensor integrals using standard techniques to obtain the following result:

$$
\begin{aligned}
& \int \frac{d^{D} \ell_{2}}{(2 \pi)^{D}} \frac{\ell_{2}^{\mu} \ell_{2}^{\nu}}{\left(\ell_{2}^{2}-\lambda_{\mathrm{IR}}\right)^{2}} \int d \operatorname{LIPS}_{\ell_{1}} Y_{\mu \nu}\left(\ell_{1}\right) \\
& =-\frac{i \Gamma(-1+\epsilon)}{2(4 \pi)^{2-\epsilon}}\left(\lambda_{\mathrm{IR}}\right)^{1-\epsilon} \int d \operatorname{LIPS}_{\ell_{1}} Y_{\mu}^{\mu}\left(\ell_{1}\right),
\end{aligned}
$$

where $\epsilon=(4-D) / 2, Y$ contains the rest of the trace in Eq. (12), and terms linear in $\ell_{2}$ cancel. Since the phasespace integral can, at worst, result in a $\log \left(\lambda_{\mathrm{IR}}\right)$ divergence, the factor $\left(\lambda_{\mathrm{IR}}\right)^{1-\epsilon}$ ensures that the expression goes smoothly to zero as $\lambda_{\mathrm{IR}}$ approaches zero for all orders in $\epsilon$. Therefore, the cut vanishes, along with the UV anomalous dimension.

Conclusions. - We have derived a new nonrenormalization theorem that applies to higher-dimensional operators in quantum field theory. Since the theorem is dependent on only the number and type of fields in each operator, it applies to generic massless theories with no relevant operators.

Besides being helpful to find zeros of the anomalousdimension matrix, the on-shell formalism of Ref. [11] is a good way to compute nonzero entries as well. Whenever an entry is excluded by our theorem, it should be much simpler to compute the entry at the next loop order compared to computing a generic entry at that loop order, because only tree-level quantities enter the cuts. In addition, helicity selection rules [7] might then apply, pushing the zero one loop further. For instance, it is straightforward to confirm that many of the nonzero entries in the tables above vanish in the absence of Yukawa couplings. It would also be interesting to combine our results with those of Ref. [18], where dimensional-analysis counting rules are used to constrain coupling-constant dependence and, more generally, to find the full set constraints in the multiloop anomalous-dimension matrix of the SMEFT. On-shell methods [9] are also a good way to compute amplitudes including higher-dimension operators. Using these, we have computed four-point one-loop massless amplitudes and associated anomalous dimensions of the SMEFT dimension-six operators, which will be described elsewhere [17].

We thank Clifford Cheung, Enrico Herrmann, Aneesh Manohar, Ian Moult, Chia-Hsien Shen, and George Sterman for very helpful discussions. We thank the U.S. Department of Energy (DOE) for support under Grant No. DE-SC0009937. We are also grateful to the Mani L. Bhaumik Institute for Theoretical Physics for generous support.

[1] W. Buchmuller and D. Wyler, Nucl. Phys. B268, 621 (1986).

[2] B. Grzadkowski, M. Iskrzynski, M. Misiak, and J. Rosiek, J. High Energy Phys. 10 (2010) 085.

[3] I. Brivio and M. Trott, Phys. Rep. 793, 1 (2019).

[4] C. Grojean, E. E. Jenkins, A. V. Manohar, and M. Trott, J. High Energy Phys. 04 (2013) 016; J. Elias-Miró, J. R. Espinosa, E. Masso, and A. Pomarol, J. High Energy Phys. 08 (2013) 033; 11 (2013) 066.

[5] E. E. Jenkins, A. V. Manohar, and M. Trott, J. High Energy Phys. 10 (2013) 087; 01 (2014) 035; R. Alonso, E. E. 
Jenkins, A. V. Manohar, and M. Trott, J. High Energy Phys. 04 (2014) 159.

[6] R. Alonso, E. E. Jenkins, and A. V. Manohar, Phys. Lett. B 739, 95 (2014); J. Elias-Miró, J. R. Espinosa, and A. Pomarol, Phys. Lett. B 747, 272 (2015).

[7] C. Cheung and C. H. Shen, Phys. Rev. Lett. 115, 071601 (2015).

[8] M. L. Mangano and S. J. Parke, Phys. Rep. 200, 301 (1991).

[9] Z. Bern, L. J. Dixon, D. C. Dunbar, and D. A. Kosower, Nucl. Phys. B425, 217 (1994); B435, 59 (1995); Z. Bern and A. G. Morgan, Nucl. Phys. B467, 479 (1996); Z. Bern, L. J. Dixon, and D. A. Kosower, Nucl. Phys. B513, 3 (1998); R. Britto, F. Cachazo, and B. Feng, Nucl. Phys. B725, 275 (2005).

[10] A. Brandhuber, M. Kostacinska, B. Penante, G. Travaglini, and D. Young, J. High Energy Phys. 08 (2016) 134; Phys. Rev. Lett. 119, 161601 (2017); J. High Energy Phys. 12 (2018) 076; 12 (2018) 077.

[11] S. Caron-Huot and M. Wilhelm, J. High Energy Phys. 12 (2016) 010.
[12] Z. Bern, C. Cheung, H. H. Chi, S. Davies, L. Dixon, and J. Nohle, Phys. Rev. Lett. 115, 211301 (2015); Z. Bern, H. H. Chi, L. Dixon, and A. Edison, Phys. Rev. D 95, 046013 (2017).

[13] G. F. Sterman, Phys. Rev. D 17, 2773 (1978); 17, 2789 (1978).

[14] L. Magnea and G. F. Sterman, Phys. Rev. D 42, 4222 (1990); W. T. Giele and E. W. N. Glover, Phys. Rev. D 46, 1980 (1992); Z. Kunszt, A. Signer, and Z. Trocsanyi, Nucl. Phys. B420, 550 (1994); S. Catani, Phys. Lett. B 427, 161 (1998).

[15] G.F. Sterman, An Introduction to Quantum Field Theory (Cambridge University Press, Cambridge, England, 1993).

[16] J.-y. Chiu, A. Fuhrer, R. Kelley, and A. V. Manohar, Phys. Rev. D 80, 094013 (2009).

[17] Z. Bern, J. Parra-Martinez, and E. Sawyer (to be published).

[18] E. E. Jenkins, A. V. Manohar, and M. Trott, Phys. Lett. B 726, 697 (2013). 